\title{
An elastomeric grating coupler
}

To cite this article: Askin Kocabas et al2006 J. Opt. A: Pure Appl. Opt. 885

View the article online for updates and enhancements.

\section{Related content}

Fabrication of reverse symmetry polymer wavequide sensor chips

Robert Horvath, Henrik C Pedersen, Nina

Skivesen et al.

Fabrication of all-polymer freestanding wavequides

Róbert Horváth, Lars R Lindvold and Niels

B Larsen

Mirror-based surface optical input/output technology with precise and arbitrary coupling angle for silicon photonic application

Akihiro Noriki, Takeru Amano, Masahiko Mori et al.

\section{Recent citations}

- $\frac{\text { Reusable thin-film all-polymer couplers for }}{\text { vertical light coupling to single-mode }}$
$\frac{\text { waveguides }}{\text { Pei Li et al }}$
- Conventional elastomers doped with
$\frac{\text { benzophenone derivatives as effective }}{\text { media for all-optical fabrication of tunable }}$
$\frac{\text { diffraction elements }}{\text { Alexander Ryabchun et al }}$
- Grating coupler integrated photodiodes for
plasmon resonance based sensing
Burak Turker et al




\title{
An elastomeric grating coupler
}

\author{
Askin Kocabas, Feridun Ay, Aykutlu Dâna and Atilla Aydinli ${ }^{1}$ \\ Türk Telekom Bilkent Laboratory, Department of Physics, Bilkent University, 06800 Ankara, \\ Turkey \\ E-mail: aydinli@fen.bilkent.edu.tr
}

Received 19 August 2005, accepted for publication 17 November 2005

Published 13 December 2005

Online at stacks.iop.org/JOptA/8/85

\begin{abstract}
We report on a novel nondestructive and reversible method for coupling free space light to planar optical waveguides. In this method, an elastomeric grating is used to produce an effective refractive index modulation on the surface of the optical waveguide. The external elastomeric grating binds to the surface of the waveguide with van der Waals forces and makes conformal contact without any applied pressure. As a demonstration of the feasibility of the approach, we use it to measure the refractive index of a silicon oxynitride film. This technique is nondestructive, reversible, low cost and can easily be applied to the characterization of optical materials for integrated optics.
\end{abstract}

Keywords: grating coupler, PDMS

\section{Introduction}

Two common methods use prisms and gratings for coupling free space light into an optical waveguide. In the prism coupling approach, adjustment of the gap between the prism and the waveguide is critical and requires significant pressure, with the associated possibility of damage to the waveguide or the prism [1,2]. Also, the bulky nature of the prism makes it unsuitable for applications in integrated devices. Finally, coupling to high index waveguides requires the use of expensive higher index prisms. Due to their compact size and planarity, the grating couplers are superior to prism couplers in these respects. The idea of the grating coupler goes back to the work of Dakss et al [3]. The grating coupler is based on the periodic modulation of the refractive index on the surface of the waveguide. There are several techniques for fabricating gratings. Since for most applications submicron periodicity is required, interference or electron-beam lithographies [4] are used to place the required pattern on the surface, followed by wet or dry etching of the surface relief. Many varieties of grating couplers on various materials have, over the years, been designed and fabricated. Grating couplers have been etched on semiconductors [5] and dielectric waveguides [6] as well as onto polymeric waveguides [7]. Once the grating is manufactured on the waveguide, it is impossible to reconfigure or remove the grating without damaging the waveguide. These factors restrict the use of grating couplers for testing of planar waveguides on a routine basis. The need for a grating coupler

1 Author to whom any correspondence should be addressed. that can be fabricated with relatively low cost and can be applied reversibly to many optical waveguides for coupling light remains.

In this paper, we describe a new approach for using grating couplers to couple free space light into optical waveguides that overcomes all of these problems. To demonstrate the feasibility of the approach, we measure the refractive index of a well characterized silicon oxynitride ( $\mathrm{SiON}$ ) waveguide. We create an effective index perturbation using an external and removable elastomeric grating. We fabricate the elastomeric diffraction grating from polydimethylsiloxane (PDMS) whose bulk refractive index is 1.41 [8], using replica moulding techniques. This technique was extensively used in the fabrication of polymer distributed feedback lasers [9], micromoulding of polymer in capillaries [10] and in polymer Mach-Zehnder modulators [11]. There have been several previous works on PDMS gratings. Rogers et al [12] have reported a photothermal detector using a PDMS diffraction grating. Grzybowski et al [13] used mechanical stress to deform a PDMS grating for applications in modulators and force sensors. PDMS gratings were also used for microfluidic [14] and pressure sensing [15] applications. However, this is the first time that a PDMS grating has been used as an optical coupler and used to measure the refractive index of slab waveguides.

\section{Experimental details}

The replication procedure is shown in figure 1 . The first step is the fabrication of a master grating. A $1 \mathrm{~cm}^{2}$ master 


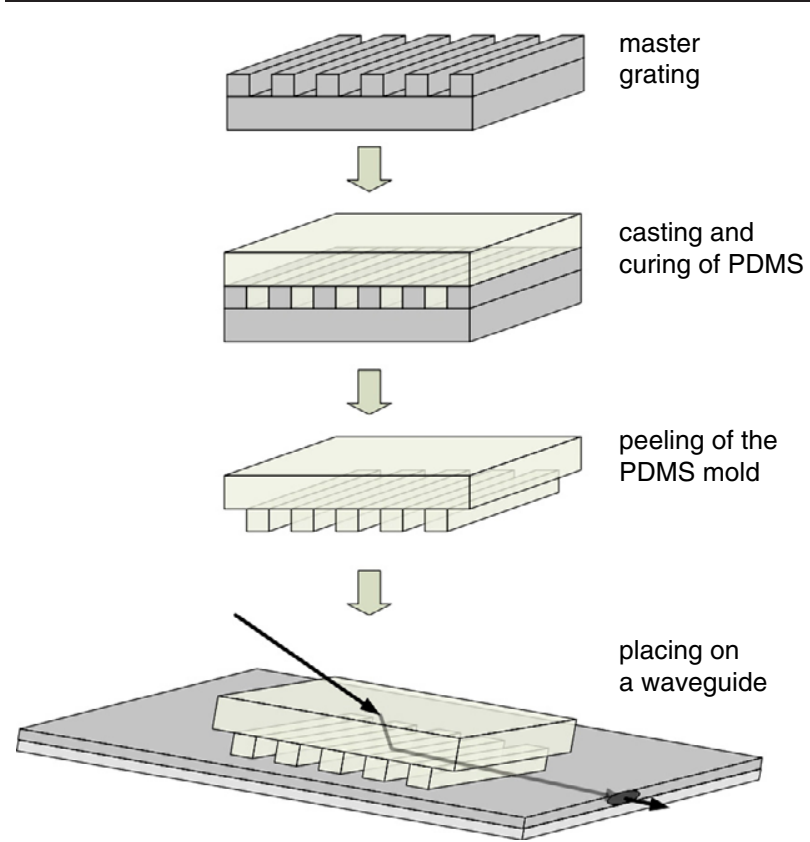

Figure 1. Schematic illustration of elastomeric grating coupler fabrication steps.

(This figure is in colour only in the electronic version)

grating with a rectangular profile is fabricated on a silicon wafer using interference lithography. The period of the grating is designed to be $600 \mathrm{~nm}$. Liquid PDMS (Sylgard 184) is then poured on the master grating and a polished wafer was placed on the top surface with rigid separators in order to fabricate planar and smooth surfaces, and cured at $70^{\circ} \mathrm{C}$ for $3 \mathrm{~h}$ in air. The PDMS stamp is then peeled off from the master grating. The thickness of the PDMS grating stamp is $5 \mathrm{~mm}$. After peeling, the grating stamp is placed on the slab waveguide. The PDMS allows conformal contact between the stamp and the support. Sinusoidal, triangular and rectangular profiles were investigated. Submicron features on gratings with triangular and sinusoidal profiles were occasionally observed to collapse during conformal contact with the waveguide surface [16]. However, the grating with the rectangle profile where the grating groove depth was $200 \mathrm{~nm}$ gave excellent results. It should be noted that, due to van der Waals interaction between the elastomeric stamp and the waveguide, pressure is not required to keep the stamp on the waveguide. In fact, a PDMS stamp can stick on a vertical silicon wafer surface without sag. A wetting front initiated due to van der Waals forces progresses across the surface due to the low free surface energy of the PDMS [17] to form a self-supported intimate contact.

Resonant coupling between the light source and the waveguide occurs when the wavevector matching condition is satisfied. The wavevector matching condition can be calculated using the well known grating equation

$$
k_{f, i}=k_{0} \sin \left(\theta_{i}\right)+m \frac{2 \pi}{\Lambda},
$$

where $k_{0}$ is the wavevector of the incoming light with incidence angle $\theta_{i}, k_{f, i}$ is the wavevector of the $i$ th available mode of the guiding film, $\Lambda$ is the period of the grating structure and $m$ is an integer that defines the order of the scattering process. $k_{0} \sin \left(\theta_{i}\right)$ is the horizontal component of the wavevector.

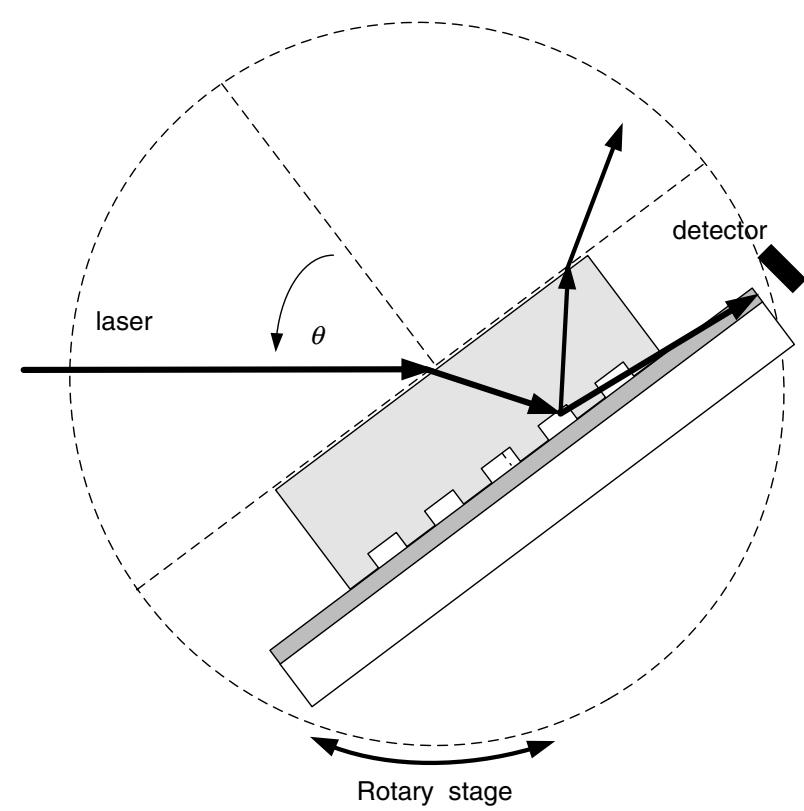

Figure 2. A schematic illustration of the experimental set-up allowing rotation of the sample so as to satisfy the resonance coupling.

It should be noted that, since the top surface of the PDMS grating stamp is parallel to the contact interface, the wavevector matching condition on the top surface holds for the PDMS stamp and waveguide interface as well, eliminating the need to precisely know the refractive index of PDMS.

The experimental set-up is shown in figure 2. The grating is placed on the slab waveguide whose refractive index is to be measured and both are mounted on a rotary stage. The waveguide films were grown by the standard PECVD technique using silane, nitrous oxide and ammonia as precursor gases. The refractive index of the $\mathrm{SiON}$ slab waveguide was measured with a prism coupling set-up with a precision of $\pm 2 \times$ $10^{-4}$. The sample is illuminated with a HeNe laser operating at a wavelength of $632.8 \mathrm{~nm}$ and the angle of incidence is scanned using a high resolution motorized stage with step size of $0.005^{\circ}$ under computer control. The alignment of the grating grooves with the plane of incidence is done by making use of the 0 th-and -1 th-order diffracted beams. The zero of the angle of incidence is calibrated by aligning the incident beam with the back-reflected beam. The experiment was performed for both transverse magnetic (TM) and transverse electric (TE) polarizations. At specific incident angles, depending on the index of refraction and the thickness of the slab waveguide as well as the period of the grating stamp, the incident light couples into the slab waveguide. Once coupled, light is guided in the slab waveguide and detected at the end of the waveguide with a large area silicon photodetector. It is, however, possible to out-couple the light via a second grating as well.

\section{Results and discussion}

The measurement results are shown in figure 3. Several sharp peaks show the coupling angles to the modes propagating in the waveguide for both TE and TM polarizations. Due to the geometrical birefringence of the slab waveguide, there 


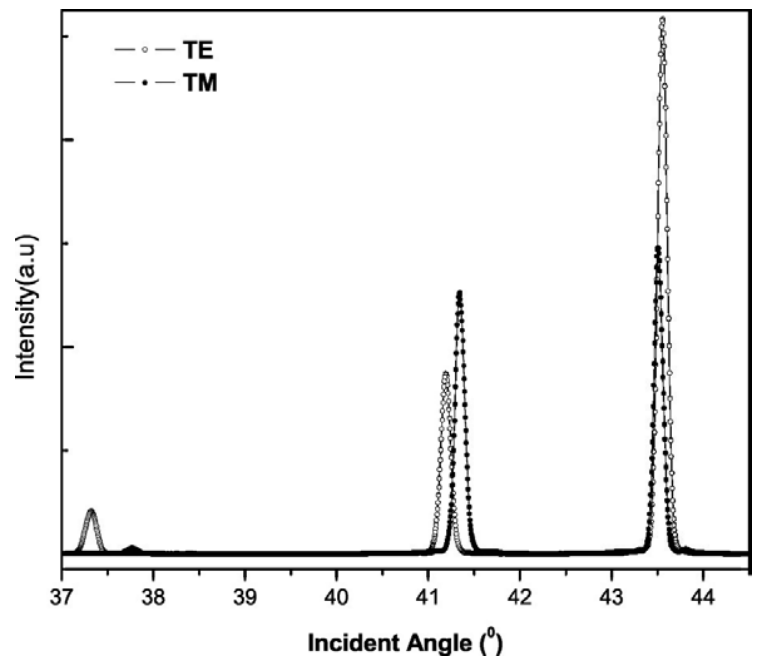

Figure 3. Detected intensity as a function of the coupling angle for both TE and TM modes.

is a small shift between TE and TM coupling angles. The period of the grating stamp was determined by measuring the position of the diffracted beams from the PDMS grating stamp used in the transmission mode. The period of the master grating was designed to be $600 \mathrm{~nm}$, but due to the thermal contraction, the periodicity of the elastomeric stamp was found to change from the designed $600 \mathrm{~nm}$ down to $594.5 \pm 0.1 \mathrm{~nm}$. Using equation (1), the effective indices for each mode were calculated. The calculations were done by first solving the Maxwell equations with the corresponding boundary conditions. Then, using the measured effective indices for the guided modes, the thickness and bulk refractive index of the slab waveguide were calculated. The bulk refractive index and the thickness of the waveguide core were $1.745 \pm 0.001$ and $1.68 \pm 0.1 \mu \mathrm{m}$, respectively. An error analysis taking into account the uncertainties associated with the rotary stage and the period of the PDMS grating stamp shows that refractive indices can be determined with a precision of the order of $10^{-4}$ which is comparable with that obtained from prism coupling techniques. The accuracy of this method is restricted by several factors. Measurement of the grating period has an uncertainty of $\pm 0.1 \mathrm{~nm}$, resulting in $\Delta n=$ $\pm 4 \times 10^{-4}$. The angular resolution of the rotation stage is $0.005^{\circ}$ which gives $\Delta n= \pm 1 \times 10^{-4}$; this is in accordance with the results obtained for bulk index calculations of this study.

In a lot of applications of the grating couplers, the figure of merit is the efficiency of the coupling. The coupling efficiency depends on the grating parameters such as the groove shape, beam waist and beam position on the grating [18]. In our measurement set-up, we focus the laser beam and decrease the beam waist. This small beam waist decreases the coupling efficiency making it of the order of $1 \%$. While this is more than sufficient for our purposes, the efficiency can be increased to the theoretical value of $81 \%$ [19] by increasing the beam waist, optimizing the beam position and using a higher refractive index elastomer. On the other hand, there are a number of applications where efficiency of coupling is not a major requirement. PDMS elastomeric gratings can be used in applications such as measurements of refractive indices, or as out-couplers for signal pickup.

\section{Conclusions}

In conclusion, we introduced a new, inexpensive, nondestructive and reversible technique for coupling light to guided modes of an optical waveguide. This approach eliminates the need for extensive sample preparation and makes the measurement of the refractive index of the slab waveguide very simple. Unlike the prism coupling method where pressure has to be applied to adjust the air gap to couple the light into the waveguide, no pressure is needed in our approach. This eliminates the possibility of stress induced artefacts in the measurement of refractive indices. This is especially important in the refractive index measurements of soft materials such as some polymers [2]. We applied this technique to measure the refractive index of $\mathrm{SiON}$ slab waveguides with a precision of the order of $10^{-4}$. The advantages of this approach make it superior over others for characterization of optical waveguides. Finally, due to the fact that the PDMS grating can easily be removed and reoriented along any crystallographic direction, this method provides a very convenient way to measure refractive indices of birefringent materials.

\section{Acknowledgments}

The authors gratefully acknowledge the financial support of NATO Scientific Programme under grant number PST.NR.CLG 980588.

\section{References}

[1] Tien P K 1971 Appl. Opt. 102395

[2] Ay F, Kocabas A, Kocabas C, Aydinli A and Agan S 2004 J. Appl. Phys. 967147

[3] Dakss M L, Kuhn L, Heidrich P F and Scott B A 1970 Appl. Phys. Lett. 16523

[4] Tennant D M, Koch T L, Mulgrew P P and Gnall R P 1992 J. Vac. Sci. Technol. B $\mathbf{1 0} 2530$

[5] Hagberg M, Eriksson N and Larsson A 1995 Appl. Phys. Lett. 673685

[6] Fattinger Ch 1993 Appl. Phys. Lett. 621460

[7] Waldhäusl R, Schnabel B, Dannberg P, Kley E, Bräuer A and Karthe W 1997 Appl. Opt. 369383

[8] Vezenov D V, Mayers B T, Wolfe D B and Whitesides G M 2005 Appl. Phys. Lett. 86041104

[9] Rogers J A, Meier M, Dodabalapur A, Laskowski E J and Cappuzzo M A 1999 Appl. Phys. Lett. 743257

[10] Kim E, Xia Y and Whitesides G M 1995 Nature 367581

[11] Palaczi G T, Huang Y and Yariv A 2004 Appl. Phys. Lett. 851662

[12] Rogers J A, Jackman R J, Schueller O J A and Whitesides G M 1996 Appl. Opt. 386641

[13] Grzybowski B, Qin D, Haag R and Whitesides G M 2000 Sensors Actuators A 8681

[14] Schueller O J A, Zhao X M, Whitesides G M, Smith P and Prentiss M 1999 Adv. Mater. 1137

[15] Hosokawa K, Hanada K and Maeda R 2002 J. Micromech. Microeng. 121

[16] Delamarche E, Schmid H, Michel B and Biebuyck H 1997 Adv. Mater. 9741

[17] Sundar V C, Zaumseil J, Podzorov V, Menard E, Willett R L, Someya T, Gershenson M E and Rogers J A 2004 Science 3031644

[18] Pascal D, Orobtchouk R, Layadi A, Koster A and Laval S 1997 Appl. Opt. 362443

[19] Dalgoutte D G 1973 Opt. Commun. 8124 